DOI: https://doi.org/10.46296/gt.v3i5.0012

\title{
TUBERCULOSIS EXTRAPULMONAR: PRESENTACIÓN DE UN CASO CLÍNICO
}

\section{EXTRAPULMONARY TUBERCULOSIS: PRESENTATION OF A CLINICAL CASE}

\author{
Echeverria-Pilco María José ${ }^{*}$ \\ ${ }^{1}$ Universidad Laica Eloy Alfaro de Manabí, ULEAM. Manta, Ecuador.
}

*Correo: auglo1971.10@hotmail.com

\section{Resumen}

La tuberculosis abdominal comprende alrededor del $5 \%$ de todos los casos de tuberculosis, esta patología es considerada una enfermedad de inicio insidioso y presentación crónica, misma que rara vez es catalogada como una urgencia; sus complicaciones, sin un adecuado enfoque clínico, pueden desorientar al médico, en muchos casos de tuberculosis en niños, la confirmación de laboratorio nunca se establece, como sucede con este caso en particular y es allí donde se determina que un diagnóstico presuntivo de tuberculosis abdominal basado en la respuesta clínica o imagenológica que presente el paciente al tratamiento empírico, especialmente para situaciones en las que existe un alto índice de sospecha de esta patología.

Palabras clave: tuberculosis abdominal, tuberculosis en niños, tratamiento.

\begin{abstract}
Abdominal tuberculosis comprises about 5 percent of all cases of Tuberculosis, this pathology is considered an insidious onset disease and chronic presentation, which is rarely listed as an emergency, its complications, without an adequate clinical approach, can misleading the doctor, in many cases of tuberculosis in children, laboratory confirmation is never established, as is the case with this particular case and it is there that we can establish a presumptive diagnosis of abdominal tuberculosis based on the clinical or imaging response presented by the patient to empirical treatment, especially for situations in which there is a high index of suspicion of this pathology.
\end{abstract}

Keywords: abdominal tuberculosis, tuberculosis in children, treatment.

Información del manuscrito:

Fecha de recepción: 12 de septiembre de 2019

Fecha de aceptación: 25 de noviembre de 2019

Fecha de publicación: 10 de enero de 2020 


\section{Introducción}

La tuberculosis de localización intestinal $\mathrm{y} / \mathrm{o}$ peritoneal es un padecimiento crónico cuyos síntomas y signos son inespecíficos. La tuberculosis digestiva con sus variantes: intestinal, peritoneal, enteroperitoneal, mesentérica con o sin compromiso de otros órganos puede imitar y semejar a una gran variedad de desórdenes abdominales, incluyendo patología neoplásica, infecciosa e inflamatoria inespecífica. Un evidente grado de agudeza clínica es importante para el diagnóstico (Tirado et al., 2003).

En la tuberculosis intestinal el síntoma frecuente es el dolor abdominal, tanto espontáneo como a la palpación, es de localización difusa, a predominio del cuadrante inferior derecho y se acompaña generalmente de anorexia y náuseas. Existe alteración del patrón defecatorio con presencia de diarreas persistentes o alternancia de diarrea con estreñimiento, las heces pueden ser acuosas o de tipo disentérico con presencia de moco y sangre. La hematoquecia puede presentarse en la forma ulcerosa. Signos importantes son la pérdida de peso, la distensión abdominal, fiebre, y borborigmos en la fosa ilíaca derecha; no obstante, también, es posible palpar masa dolorosa en el cuadrante inferior derecho (GómezZuleta et al., 2012).

Los factores de riesgo que se atribuyen al desarrollo de tuberculosis extrapulmonar son fundamentalmente la edad, el sexo femenino, la existencia de infección por $\mathrm{VIH}$ y las comorbilidades del paciente como la presencia de insuficiencia renal crónica, diabetes mellitus o la existencia de inmunodepresión (Ramírez-Lapausa et al., 2015).

Establecer el diagnóstico requiere un elevado índice de sospecha. EI retraso en el diagnóstico de las formas extrapulmonares es un hecho frecuente que conlleva un aumento de morbilidad y mortalidad. Los síntomas y signos pueden ser inespecíficos y en ocasiones se presenta en pacientes con radiografía de tórax y baciloscopia de esputo negativa, lo que dificulta que se tenga en consideración en el diagnóstico inicial. Aún así siempre se debe descartar la presencia de tuberculosis pulmonar mediante radiología y cultivo de esputo. Se debe realizar de prueba de 
tuberculina (PT) o test de detección de interferón gamma para descartar la presencia de infección tuberculosa (Sandrino-Sánchez et al., 2015).

Las técnicas basadas en la detección del interferón gamma en sangre (IGRA), ayudan en el diagnóstico, sin embargo, su ausencia no excluye la posibilidad de enfermedad tuberculosa extrapulmonar, ya que pueden obtenerse resultados negativos hasta en el $68 \%$ de los casos. En las formas extrapulmonares de tuberculosis, la dificultad para la obtención de muestras microbiológicas hace que la radiología y otras técnicas de imagen como TC o Resonancia magnética puedan ser de gran ayuda en la aproximación diagnóstica y para la obtención de muestras mediante punción.

La tinción para detectar la presencia de bacilos ácido-alcohol resistente (BAAR), mediante el empleo de las técnicas de Ziehl-Neelsen, permite realizar un diagnóstico rápido. No obstante, para que sean detectables deben existir entre 5000-10000 bacilos/mL en la muestra. Es por ello que el rendimiento de la baciloscopia en las presentaciones de tuberculosis extrapulmonares es más elevado en muestras obtenidas de tejidos por biopsia (sensibilidad $>70-80 \%$ ), que en aquellas muestras de líquidos biológicos (5-20\%). Se debe tener en cuenta que un porcentaje variable (30-50\%) de los casos de tuberculosis extrapulmonar pueden tener una baciloscopia negativa. Por tanto, el objetivo del presente manuscrito es describir la evolución de una paciente con diagnóstico de tuberculosis extrapulmonar.

\section{Metodología}

\subsection{Caso clínico}

El caso de estudio corresponde a un paciente de 14 años de edad, de sexo femenino, procedente de zona rural y consideración étnica mestiza. Entre sus antecedentes patológicos personales presenta bajo peso al nacer, cardiopatía congénita: comunicación interventricular, insuficiencia tricúspidea moderada, insuficiencia pulmonar leve e hipertensión pulmonar moderada, misma que fue diagnosticada a los 8 años, con criterio para resolución quirúrgica que no se realiza por negatividad de los progenitores. 
La paciente acudió a emergencia por presentar cuadro clínico de 1 mes de evolución, caracterizado por dolor abdominal difuso, deposiciones diarreicas de consistencia líquida: 3 a 4 ocasiones al día con moco, acompañada de alza térmica no cuantificada persistente, vómitos: 2 a 3 ocasiones al día y malestar general. En las dos últimas semanas presenta tos que moviliza secreciones, y se intensifica hace 3 días.

Presenta el antecedente de recibir tratamiento con trimetropin sulfametoxazol durante 7 días y paracetamol sin presentar mejoría clínica, además, refiere un contacto hace tres meses, con abuela materna que tiene antecedente de tuberculosis pulmonar bacilífera, en aquella fecha no se realizó ningún estudio ni tratamiento.

\subsubsection{Examen físico}

Presenta un peso de $34 \mathrm{Kg}$ (índice Z3); talla: $144 \mathrm{~cm}$ (índice Z-3); Imc: 16,4 (índice Z-3).

Frecuencia cardíaca: 131 latidos por minuto; frecuencia respiratoria: 23 respiraciones por minuto; temperatura: $38,4^{\circ} \mathrm{C}$; saturación de oxígeno: 96\%; tensión arterial: $100 / 70 \mathrm{mmHg}$.

Se trata de una paciente con facies álgica, poco colaboradora con la anamnesis. Se detectaron mucosas semihúmedas; llenado capilar: 3segundos, no se presentan nódulos subcutáneos.

Sistema osteoarticulomuscular: no presenta patología asociada.

Cabeza: fosas nasales permeables. Boca simétrica, mucosas semihúmedas.

Cuello: sin patología asociada.

Tórax-Aparato respiratorio: campos pulmonares con murmullo vesicular disminuido. Presencia de roncus en zona bilateral.

Aparato circulatorio: Soplo sistólico en válvula aórtica G IV, pulsos periféricos presentes y simétricos.

Abdomen: distendido, doloroso a la palpación superficial y profunda. Se detecta hepatomegalia $4 \mathrm{~cm}$ por debajo del reborde costal. Se palpa esplenomegalia. Ruidos hidroaéreos (RHA) positivos y propulsivos.

Extremidades: sin patología. 


\subsubsection{Pruebas complementarias y evolución}

Hemograma a su ingreso

Hemoglobina $(\mathrm{Hb}): \quad 7,6 \quad \mathrm{~g} / \mathrm{L}$, hematocrito: $21,2 \%$, plaquetas $43 \mathrm{x}$

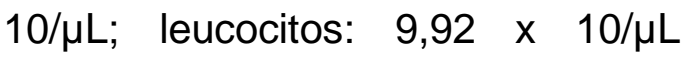
(Linfocitos 8,3\%; Neutrófilos 88\%); MCV: $74,9 \mathrm{fl}, \mathrm{MCH}: 26,9 \mathrm{fl}, \mathrm{MCHC}$ : 35,8 g/dL; glucosa: $106 \mathrm{mg} / \mathrm{dL}$, creatinina: $0,94 \mathrm{mg} / \mathrm{dL}$, sodio: 138,6 $\mathrm{mEq} / \mathrm{L}$, potasio: $3,12 \mathrm{mEq} / \mathrm{L}$, cloro: 106,6 mEq/L. El estudio Elemental microscópico de orina (EMO) fue normal.

Rx de tórax: índice cardiotorácico de 0,7 , aumento de la trama broncopulmonar, infiltrado paracardíaco y basal derecho. Presenta deposiciones líquidas con moco sin presencia de sangre, dolor abdominal, artralgias, tos con expectoración de aproximadamente 2 semanas de evolución y disnea de mediano esfuerzo; se prescribe hidratación parenteral con cloruro de sodio $0,9 \%, 80 \mathrm{~mL} / \mathrm{h}$, ampicilina + sulbactam 1,5gr intravenoso cada 6 horas, paracetamol en caso de fiebre.

\section{Hemograma día 2}

Hemoglobina $(\mathrm{Hb}): \quad 8 \mathrm{~g} / \mathrm{L}$, hematocritos: $22,1 \%$, plaquetas: $36 \mathrm{x}$

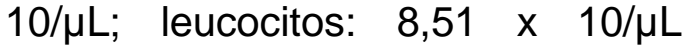
(Linfocitos 6,3\%; Neutrófilos 90,6\%); MCV: $75,4 \mathrm{fl} ; \mathrm{MCH}: 27,3 \mathrm{fl}$ y MCHC: $36,2 \mathrm{~g} / \mathrm{dL}$.

TGO: 36,9 g/dL; TGP: 43,63 y 5,8 g/dL.

VIH (4ta generación): no reactivo.

Presenta abdomen distendido, extremidades con edema, alza térmica de $39,7^{\circ} \mathrm{C}$; a pesar de la presencia de trombocitopenia no se evidencian petequias ni hematomas, se prescribe hidratación parenteral de mantenimiento de la dextrosa al $5 \%$ en agua + cloruro de sodio $0,9 \%$ + sodio $30 \mathrm{meq}+$ potasio $20 \mathrm{meq} \mathrm{a}$ $40 \mathrm{~mL} / \mathrm{h}$; se suspende ampicilina + sulbactam, se inicia el tratamiento con oxacilina 2gr cada 6 horas, score downes cada 6 horas.

Se pasa paquete globular; al término de la transfusión sanguínea se prescribe furosemida $10 \mathrm{mg}$.

\section{Hemograma día 3}

Post transfusión. Hemoglobina $(\mathrm{Hb})$ : $11 \mathrm{~g} / \mathrm{L}$, hematocritos: 31,2\%; plaquetas: $64 \times 10 / \mu \mathrm{L}$; leucocitos:

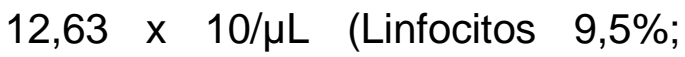
Neutrófilos 85,8\%); MCV: $78 \mathrm{fl}, \mathrm{MCH}$ : 27,6 fl, MCHC: $35,3 \mathrm{~g} / \mathrm{dL}$; glucosa: $103 \mathrm{mg} / \mathrm{dL}$, creatinina: 0,88 mg/dL; 
sodio: $137,8 \mathrm{mEq} / \mathrm{L}$; potasio: 3,17 $\mathrm{mEq} / \mathrm{L}$, cloro: 108,9 mEq/L.

Gasometría arterial

$\mathrm{pH}: 7,4 ; \mathrm{PCO}_{2}: 21,8 ; \mathrm{PO}_{2}: 100$; $\mathrm{TCO}_{2}: 14.6 ; \mathrm{HCO}_{3}: 14 ; \mathrm{SO}_{2}: 98$.

Paciente con alza térmica $38,7^{\circ} \mathrm{C}$, dolor a la palpación en hipogastrio y área pélvica, dolor poliarticular, hidratación parenteral: dextrosa al $5 \%$ en agua + cloruro de sodio $40 \mathrm{mEq}+$ cloruro de potasio $45 \mathrm{mEq}$, se continúa con indicación de oxacilina antes indicada, se prescribe ranitidina $50 \mathrm{mg}$ cada día, tramadol $45 \mathrm{mg}$ en $50 \mathrm{~cm}^{3}$ de solución salina al $0,9 \%$ Stat, paracetamol $675 \mathrm{mg}$ intravenoso cada 6 horas, ceftriaxona $2 \mathrm{gr}$ intravenosos cada 12 horas, metronidazol $380 \mathrm{mg}$ intravenoso cada 8 horas.

\section{Hemograma día 4}

Hemoglobina $(\mathrm{Hb}): \quad 11,2 \quad \mathrm{~g} / \mathrm{L}$, hematocritos: $31,6 \%$, plaquetas: $71 \mathrm{x}$

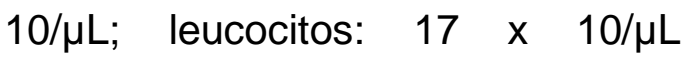
(Linfocitos 8,3\%; Neutrófilos 85.2\%); MCV: $77 \mathrm{fl}, \mathrm{MCH}: 27,5 \mathrm{fl}, \mathrm{MCHC}$ : $35,4 \mathrm{~g} / \mathrm{dL}$. Reticulocitos: 1,4\%.

Frotis: Hipocromía, Anisocitosis, Poiquilocitosis, PLT: $75.000 \mathrm{~mm}^{3}$.
Creatinina: $0,65 \mathrm{mg} / \mathrm{dL}$, bilirrubina total: $0,51 \mathrm{mg} / \mathrm{dL}$; bilirrubina directa: $0,17 \mathrm{mg} / \mathrm{dL}$, bilirrubina indirecta: 0,34 mg/dL, TGO: 40,2 U/L, TGP: 37,9 U/L; LDH: 413,15 u/L, PCR: $1 \mathrm{mg} / \mathrm{L}$, VSG: $17 \mathrm{~mm} / \mathrm{h}$; TP: 14,7 Seg, TTP: 50,8 Seg, INR: 1,45.

HCG-BETA: 0,173 mL U/mL

Paciente con alza térmica de $40^{\circ} \mathrm{C}$, extremidades inferiores con edema, abdomen distendido, Blumberg.

\section{Día 5}

Ecografía abdominal: bazo incrementado de tamaño, mismo que mide $14 \mathrm{~cm}$; líquido libre laminar perihepático, esplenomegalia.

La ecografía pélvica evidenció que la vejiga, útero y ovarios se encuentran dentro de parámetros normales, líquido libre de moderada cantidad en espacio de Douglas. Se detectó la presencia de distensión abdominal, dolor intenso a la palpación superficial y profunda en fosa iliaca derecha e izquierda, alza térmica $38,9^{\circ} \mathrm{C}$, se mantienen las indicaciones.

\section{Día 6}

$\mathrm{KOH}$ : negativo.

Hemocultivo: negativo. 
Baciloscopia: Negativo.

Presenta alza térmica $39^{\circ} \mathrm{C}$, persiste el dolor abdominal y en el área pélvica, se mantienen las indicaciones.

\section{Día 7}

Proteínas totales: $7,85 \mathrm{~g} / \mathrm{dL}$.

Albumina: $3,53 \mathrm{~g} / \mathrm{dL}$.

Globulina: 4,32 g/dL.

Fosfatasa alcalina: $250,8 \mathrm{U} / \mathrm{L}$.

LDH: 374,67 U/L.

Presenta alza térmica, $38,5^{\circ} \mathrm{C}$, con pico más alto de $40,3^{\circ} \mathrm{C}$, a pesar de tener indicado antipirético; paciente con poca tolerancia oral, persiste dolor en área abdominal y en área pélvica. En este día se tomó una radiografía de tórax que no evidencia mejoría en relación a radiografía de tórax inicial. Se decide rotar antibióticos debido a que el cuadro clínico no presenta mejoría, y se evidencia progresión de infección a nivel pulmonar; se suspende oxacilina, ceftriaxona y metronidazol, se prescribe vancomicina $860 \mathrm{mg}$ intravenoso cada 12 horas, imipenem/cilastatina 750mg intravenoso cada 6 horas, claritromicina $330 \mathrm{mg}$ intravenoso cada 12 horas.

\section{Día 8}

Coproparasitario: $\quad$ PMN: $\quad 0 \%$ Negativo.

Gota gruesa: falciparum negativo; hematozoarios negativos.

Creatinina: 0,52 mg/dL, Ácido úrico: $21 \mathrm{mg} / \mathrm{dL}$.

Presenta alza térmica de $39,8^{\circ} \mathrm{C}$, persiste dolor abdominal y en área pélvica, distensión abdominal, se mantienen las indicaciones.

\section{Día 9}

Persiste la distensión abdominal, dolor abdominal y en área pélvica, se siguen presentando picos febriles a pesar de administración de antipirético, alza térmica de $38^{\circ} \mathrm{C}$, se solicita ANCA-ANTI DNA-ANA, CA 125 , se mantienen indicaciones.

\section{Día 10}

Del examen físico de la paciente llama la atención la presencia de ganglios inflamados en región inguinal bilateral no dolorosos, sin presencia de picos febriles, persiste dolor en área abdominal y en área pélvica.

\section{Día 11}

Hemoglobina (Hb): 9,32 $\mathrm{g} / \mathrm{L}$, hematocritos: $27,9 \%$, plaquetas: 175 
x 10/ $\mathrm{LL}$, leucocitos: $9,37 \times 10 / \mu \mathrm{L}$ (Linfocitos 14,1\%; Neutrófilos 80,6\%); MCV: $84 \mathrm{fl}, \mathrm{MCH}: 28,0 \mathrm{fl}$, MCHC: $33,3 \mathrm{~g} / \mathrm{dL}$. La paciente presenta alza térmica $39^{\circ} \mathrm{C}$, persiste el dolor en área abdominal y en área pélvica, por tanto, se mantienen indicaciones.

\section{Día 12}

Secreción traqueal: $\mathrm{KOH}$ negativo. La paciente persiste con dolor en región abdominal y región pélvica, distención abdominal, presenta alza térmica de $38^{\circ} \mathrm{C}$, se sugiere aplicar antifímicos según resultados de IGRA.

\section{Día 13}

Hemoglobina $(\mathrm{Hb}): \quad 9,3 \quad \mathrm{~g} / \mathrm{L}$, hematocritos: $29,4 \%$, plaquetas: 171 x 10/ $\mathrm{L}$, leucocitos: $8,42 \times 10 / \mu \mathrm{L}$ (Linfocitos 20,7\%; Neutrófilos $68,5 \%), M C V: 88,4 \mathrm{fl}, \mathrm{MCH}: 27,9 \mathrm{fl}$, MCHC: 31,6 g/dL; VSG: $36 \mathrm{~mm} / \mathrm{h}$.

Prueba de Combs directa: negativa. La paciente presenta alza térmica $38,6^{\circ} \mathrm{C}$, al realizar el examen físico persiste distensión abdominal, dolor en área abdominal y en área pélvica. Se mantienen las indicaciones.

\section{Día 14}

Muestra bronquial: antibiograma sin crecimiento.

ANCAc: Negativo; ANCAp: Negativo; anticuerpos anti-DNAdc: negativo; anticuerpos anti-nucleares (ANA): negativo; CA 125: 37,5 U/mL;

QuantiFERON

(IGRA):

indeterminado.

Hemocultivo:

Staphylococcus aureus resistente a oxacilina, como lo son también a las cefalosporinas, inhibidores betalactámicos y carbapenémicos.

Hemocultivo: hongos (no se obtienen colonias durante 4 días de incubación).

En la paciente persiste el dolor en área abdominal y en área pélvica, persisten picos febriles, presentando alza térmica de $38,8^{\circ} \mathrm{C}$.

Se completa esquema antibiótico sin mejoría de cuadro clínico y se inicia tratamiento con antifímico por sospecha clínica de tuberculosis peritoneal.

\subsubsection{Interconsultas}

Interconsulta a cirugía pediátrica

Se interconsulta a cirugía pediátrica por la presencia de dolor abdominal 
en región hipogastrio y fosa iliaca derecha e izquierda, Blumberg +, con ecografía abdominal se evidencia esplenomegalia $14 \mathrm{~cm}$, ecografía pélvica en la que se evidencia líquido libre en moderada cantidad en espacio de Dowglas.

La paciente es valorada por cirugía pediátrica llegando a la conclusión que no requiere de una resolución quirúrgica, se sugiere investigar esplenomegalia.

\section{Interconsulta a ginecología}

Se interconsulta a ginecología por dolor pélvico, con ecografía pélvica que evidencia líquido libre en moderada cantidad en espacio de Dowglas. La paciente es valorada por ginecología, en la que se describe menarquía a los 9 años con ciclos irregulares que duran 5 días en moderada cantidad, vida sexual activa: negativa. A la exploración física se comprueba dolor abdominal difuso la palpación, que se irradia a región genital, sínfisis del pubis con eritema vulvar ocasionado por el uso de protector genital, himen intacto. Se realiza ecografía en la que se reporta útero de características normales, ovarios con folículos pequeños, líquido en moderada cantidad en fondo de saco de
Dowglas; por lo tanto, se descarta foco ginecológico por no tener una vida sexual activa; consecuentemente, se da el alta por ginecología.

\section{Interconsulta a medicina interna}

Se interconsulta al servicio de medicina interna por fiebre de origen desconocido, trombocitopenia no especificada, presencia de esplenomegalia, con antibioticoterapia de amplio espectro sin mejoría clínica.

La paciente es valorada por el área de medicina interna en la que concluyen que por clínica y por datos de laboratorio se sugiere estudiar patologías como linfoma, enfermedad autoinmune como lupus eritematoso sistémico y por antecedente de abuela materna con tuberculosis pulmonar se solicita BAAR esputo para descartar tuberculosis. Además, se solicitan exámenes complementarios por parte de este servicio, ANA, ANTI DNA, ANA, hemocultivo y aspirado medular.

\section{Interconsulta a cardiología}

Se solicita interconsulta a cardiología por cardiopatía congénita comunicación Interventricular, en la 
que se reafirma soplo sistólico en tricúspide Grado III, insuficiencia tricúspidea moderada, insuficiencia pulmonar leve e hipertensión pulmonar moderada; se indica tratamiento y seguimiento por cardiología, se sugiere que después de superar el proceso infeccioso se realice una cirugía para el cierre de comunicación interventricular.

\section{Interconsulta a nutrición}

Se interconsulta a nutrición por bajo peso para la edad y niveles bajos de hemoglobina.

La valoración por nutrición refiere malos hábitos alimentarios en frecuencia, tipo y tiempo de la alimentación, llegando a la conclusión de una desnutrición proteicocalórica, se indica plan nutricional, consejería nutricional y seguimiento por nutrición.

\section{Resultados y discusión}

\subsection{Cardiopatía congénita}

La válvula aórtica bicúspide (BAV) puede ser una malformación aislada, en un corazón por lo demás sano, y en ocasiones se asocia con otros defectos cardiovasculares como la coartación de la aorta (estrechamiento localizado de la arteria aorta) o dilatación aórtica (Killinger \& Lewkowicz, 2014)

La BAV se asocia a la formación de aneurismas de aorta torácica y coexiste con otros defectos congénitos, siendo el más común la coartación de aorta. Otras anomalías que se pueden asociar son CIV, persistencia del ductus arterioso, CIAs y anomalías coronarias.

La VAB aislada es una entidad clínicamente relevante, no sólo por las complicaciones relacionadas con la válvula (disfunción valvular, endocarditis infecciosa), sino también por su asociación con anomalías vasculares como la dilatación aórtica.

La ecocardiografía transtorácica (ETT) es el método diagnóstico habitual de la VAB con una sensibilidad y especificidad del $92 \%$ y $96 \%$, respectivamente en válvulas no severamente calcificadas.

Esta técnica de imagen también es útil para identificar otras alteraciones anatómicas en la raíz aórtica, la parte proximal de la aorta ascendente y otras malformaciones congénitas asociadas, así como el grado de disfunción valvular. 


\subsection{Tuberculosis Extrapulmonar (Peritoneal)}

Vineet (2017) menciona que la tuberculosis abdominal comprende alrededor del $5 \%$ de todos los casos de Tuberculosis. La tuberculosis intestinal y peritoneal es una enfermedad regional, crónica, específica, generalmente secundaria a tuberculosis pulmonar avanzada, que mayormente adopta la forma localizada en el tejido linfoide ileal, con localización frecuente en íleon terminal, yeyuno-ileal, ileocecal y/o peritoneo (Yadav et al., 2017).

La presentación clínica de la Tuberculosis (TB) extrapulmonar depende del sitio de la enfermedad. Para el diagnóstico de TB extrapulmonar, las muestras para cultivo deben recolectarse de cualquier sitio donde se sospeche infección. Cada muestra debe cultivarse independientemente de los resultados del bacilo acidorresistente (BPA). Las muestras extrapulmonares más comunes incluyen sangre completa, médula ósea, muestras de tejido (como nódulos linfáticos o huesos), líquido cefalorraquídeo, orina y líquido pleural (Ramírez-Lapausa et al., 2015; Sandrino-Sánchez et al., 2015).

Por su parte, Adams \& Starke (2017) afirma lo siguiente: "el diagnóstico de TB (pulmonar o extrapulmonar) en un niño a menudo se basa en la presencia de la tríada clásica:

1. Contacto cercano reciente con un caso infeccioso.

2. Una prueba cutánea de tuberculina positiva (TST) o liberación de interferón gamma ensayo (IGRA).

3. Hallazgos sugestivos en la radiografía de tórax o el examen físico.

Todos los datos, incluida la historia completa, el examen físico y las pruebas de diagnóstico, se deben considerar cuidadosamente.

Una historia de contacto estrecho reciente con un caso de TB infeccioso es un factor crítico para realizar el diagnóstico de TB en niños (Starke, 2014; Reto-Valiente et al., 2015). Sin embargo, es posible que el adulto enfermo aún no haya sido diagnosticado, por lo que preguntar sobre contactos enfermos y facilitar la evaluación para adultos enfermos también puede acelerar el diagnóstico de los niños. Adams \& 
Starke (2017) menciona que "en muchos casos de TB en niños, la confirmación de laboratorio nunca se establece. En tales casos, se puede hacer un diagnóstico presuntivo basado en la respuesta clínica y radiográfica al tratamiento empírico".

\section{Desnutrición proteico calórica}

Durante las últimas dos décadas, la literatura médica ha elucidado la relación entre la desnutrición intrauterina durante los primeros años de vida y un alto riesgo de padecer hipertensión y obesidad en la edad adulta.

Cuando la desnutrición no conlleva a la muerte en las primeras etapas de la vida, se gatillan mecanismos adaptativos que inducen un retardo en el crecimiento lineal y una gran susceptibilidad a enfermedades infecciosas, existiendo evidencia que asocia la desnutrición in-útero o bajo peso de nacimiento con un elevado riesgo cardiovascular y diabetes mellitus 2 en la vida adulta.

\section{Anemia moderada Microcítica Hipocrómica}

La anemia es el descenso de la hemoglobina total corporal, cabe mencionar que esta enfermedad tiene una prevalencia mundial del
25\%. Los niveles normales de hemoglobina $(\mathrm{Hb})$ varían según la edad, raza, sexo, y en determinadas situaciones como embarazo, altitud, consumo de tabaco, entre otras (Pernudy-Ubau et al., 2018).

En las anemias microcíticas, generalmente hipocrómicas (anemia $\mathrm{m}-\mathrm{H}$ ), el VCM es menor a $80 \mathrm{FI}$ y la hemoglobina corpuscular media (HCM) menor a $27 \mathrm{pg} / \mathrm{dL}$. Las anemias $\mathrm{m}-\mathrm{H}$ son las más comunes en la práctica médica. La deficiencia nutricional de hierro $(\mathrm{Fe})$ y los rasgos talasémicos son las principales causas de estas anemias en pediatría (Aixalá, 2017).

Los síntomas y signos producidos por la anemia son los mismos independiente de su causa. Van a depender de la gravedad de la anemia, la velocidad de instalación y la capacidad compensatoria de los aparatos cardiovascular y respiratorio. Los síntomas son: palidez cutánea apreciada por los familiares, cansancio fácil, hipoactividad, decaimiento, cefalea, mareos, bajo rendimiento escolar.

Por su parte, los signos son: palidez cutáneo-mucosa que se aprecia en las conjuntivas, palmas de las manos, plantas de los pies y lecho 
ungueal. Taquicardia y soplos funcionales (debido al aumento del flujo sanguíneo y su turbulencia) (Pernudy-Ubau et al., 2018).

\subsection{Plan de Tratamiento:}

\section{General:}

a. Control estricto de signos vitales (curva térmica, frecuencia cardíaca, frecuencia respiratoria, tensión arterial, saturación de oxígeno)

b. Evaluar signos de deshidratación.

c. Antipirético: Paracetamol 500mg vía oral cada 6 horas

d. Medios físicos si persiste alza térmica.

e. Hidratación: Cloruro de sodio 0,9\% $1000 \mathrm{~mL}$ que deben fluir a una velocidad de $80 \mathrm{~mL} /$ hora.

Diagnóstico 1. Cardiopatía congénita: aorta válvula bicúspide + CIV.

1. Bisoprolol $2,5 \mathrm{mg}$ vía oral cada día.

2. Furosemida $20 \mathrm{mg}$ vía oral cada día.

3. Resolución quirúrgica de patología cardíaca (cierre de CIV).

Diagnóstico 2. Tuberculosis Extrapulmonar (Peritoneal).
Se utiliza esquema 2HRZE/4HR

1. Isoniazida $75 \mathrm{mg}+$ Rifampicina $150 \mathrm{mg}+$ Etambutol 275mg + Pirazinamida 400mg 2 tabletas cada día por 2 meses.

2. Isoniazida $150 \mathrm{mg}+$ Rifampicina $300 \mathrm{mg} 2$ tabletas cada día por 4 meses.

\section{Diagnóstico 3. Desnutrición} proteico calórica.

1. Dieta hipercalórica, hiperproteica distribuida en 6 tiempos.

Distribución de calorías: carbohidratos $70 \%$, cereales integrales $50 \%$, proteínas $15 \%$, fibra 30 gramos/día, grasas $15 \%$, sodio < 2,5 gramos/día.

\section{Conclusiones}

La paciente ingresó por sospechas de afección de tuberculosis peritoneal (extrapulmonar), por tanto, se realizó el tratamiento antifímico y se valoró la respuesta clínica que este presente; durante el inicio del tratamiento no se evidenció reacciones secundarias al régimen de medicamentos empleados.

Se propone un tratamiento basado en la guía de práctica clínica de Tuberculosis del Ministerio de salud Pública del Ecuador (MSP, 2018), el 
cual menciona que en niños mayores de 7 años y adultos con peso de 30 a $37 \mathrm{~kg}$ se debe administrar el esquema $2 \mathrm{HRZE} / 4 \mathrm{HR}$.

El tratamiento utilizado en tuberculosis de tipo extrapulmonar es el mismo que se emplea en casos de tuberculosis pulmonar. En el presente caso clínico se evidenció una respuesta favorable al tratamiento inicial establecido, presentando evidente mejoría clínica y en ausencia de reacción medicamentosa.

La Tuberculosis es considerada una enfermedad de inicio insidioso y presentación crónica, y aunque rara vez es una urgencia, sus complicaciones, sin un adecuado enfoque clínico, pueden desorientar al médico.

\section{Bibliografía}

Adams, L., \& Starke, J. (2017). Tuberculosis disease in children. Uptodate. Disponible en:

https://www.uptodate.com/co ntents/tuberculosis-diseasein-children

Aixalá, M. (2017). Anemia microcítica-hipocrómica: anemia ferropénica versus $b$ talasemia menor. Acta bioquímica clínica latinoamericana, 51(3), 291305.

Barriga-Angulo, G., Trejo, M. S., Rosas, A. A., Álvarez, L. L., Cruz, F. R., Hernández, M. E. M., ... \& Arumir Escorza, C. (2014). Evaluación de la prueba GeneXpert MTB/RIF en el diagnóstico rápido de la tuberculosis y de la resistencia a rifampicina en muestras extrapulmonares. Revista latinoamericana de patología clínica y medicina de laboratorio, 61(3), 140144.

Gómez-Zuleta, M. A., ViverosCarreño, D., \& Cañón, D. P. (2012). Tuberculosis intestinal: reporte de caso y revisión de la literatura. Infectio, 16(3), 178-182.

Killinger, C. R., \& Lewkowicz, J. M. (2014). Válvula aórtica bicúspide. Revista argentina de cardiología, 82(6), 569. DOI:

http://dx.doi.org/10.7775/rac.e s.v82.i6.5419

Ministerio de Salud Pública. (2018). Prevención, diagnóstico, tratamiento y control de la tuberculosis. Guía de Práctica Clínica, 2, pp. 55. Ministerio de Salud Pública. Quito, Ecuador. Obtenido de: http://salud.gob.ec

Pernudy-Ubau, A. X., CamposGómez, V. A., RojasVanegas, L. L., Ramírez, M. L., Mejía-Baltodano, G., \& 
Rodríguez-Romero,

W. (2018). Identificación de $\beta$ talasemia en anemias microcíticas hipocrómicas refractarias al tratamiento con hierro en Nicaragua. Acta Médica Costarricense, 60(4), 162-166.

Ramírez-Lapausa, M., MenéndezSaldaña, A., \& NogueradoAsensio, A. (2015). Tuberculosis extrapulmonar, una revisión. Revista española de sanidad penitenciaria, 17(1), 3-11.

Reto-Valiente, L., Pichilingue-Reto, C., Pichilingue-Prieto, O., \& Dolores-Cerna, K. (2015). Tuberculosis abdominal en niños y adolescentes. Un desafío diagnóstico. Revista de Gastroenterología del Perú, 35(4), 318-322.

Sandrino-Sánchez, M., Martínez Muñoz, M., \& Wong Díaz, L. (2015). Tuberculosis extrapulmonar. Presentación de un caso. MediSur, 13(3), 442-447.
Starke, J. (2014). Interferon-y Release Assays for Diagnosis of Tuberculosis Infection and Disease in Children. Pediatrics;134, 1763-1773.

Tirado, P. M., de Hierro Ruiz, M. L., García, R. M., Cara, J. M., Rodríguez, M. M., \& Castellano, M. C. (2003). Tuberculosis intestinal. Un reto diagnóstico. Gastroenterología hepatología, 26(6), 351-354.

Vineet, A. (2017). Abdominal tuberculosis. UpToDate.

Yadav, D. P., Madhusudhan, K. S., Kedia, S., Sharma, R., Pratap Mouli, V., Bopanna, S., ... \& Vikram, N. K. (2017). Development and validation of visceral fat quantification as a surrogate marker for differentiation of Crohn's disease and intestinal tuberculosis. Journal of gastroenterology and hepatology, 32(2), 420-426. DOI:

https://doi.org/10.1111/jgh.13 535 